\section{Control metabólico en pacientes diabéticos tipo 1 chilenos: rol del estrés psicológico}

\author{
MANUEL S. ORTIZ ${ }^{1,2, a}$, HECTOR F. MYERS ${ }^{2, b}$
}

Background: An association between psychological stress and metabolic control can occur in patients with diabetes. Aim: To determine the longitudinal association between different psychological stress domains (emotional burden, physician-related distress, regimen-related distress, diabetes-related interpersonal distress) and metabolic control of adolescents with Type 1 diabetes. Material and Methods: An intentional sample of 20 Type 1 diabetic adolescents aged $15 \pm 4$ years was followed-up for one year. Three HbAlc determinations were performed and a stress self-report was obtained with the Polonsky' Diabetes Distress Scale. Results: Several analyses were conducted using growth curve modeling. The first model included the HbAlc measures, the time term (coded as 0, 1, 2) and several covariates (age, years living with type 1 diabetes mellitus, sex, and physical activity). An overall negative linear trend was found for HbAlc. Subsequent models added the stress domains finding that high levels of emotional burden and regimen related-distress were associated with higher $\mathrm{HbA1c}$ levels across all exams. A marginal association was found between diabetes-related interpersonal distress and HbA1c. Conclusions: Stress levels in type 1 diabetic adolescents deserve attention. Different psychological stress factors predicting metabolic control trajectories in type 1 diabetic adolescents were found.

(Rev Med Chile 2014; 142: 451-457)

Key words: Adolescents; Diabetes mellitus, type 1; Stress, psychological.

I a adolescencia ha sido descrita como una etapa evolutiva marcada por grandes cambios biológicos, psicológicos y sociales ${ }^{1,2}$. Cada uno de estos cambios potencialmente puede generar altos niveles de estrés en los adolescentes. De hecho, altos niveles de estrés en adolescentes han sido reportados previamente ${ }^{3-5}$. Si a esto sumamos el diagnóstico de una enfermedad crónica como la diabetes mellitus tipo 1 (DM1), es altamente probable que adolescentes diabéticos reporten elevados niveles de estrés. En efecto, adolescentes viviendo con DM1 deben enfrentar una serie compleja de desafíos conductuales y psicológicos, las

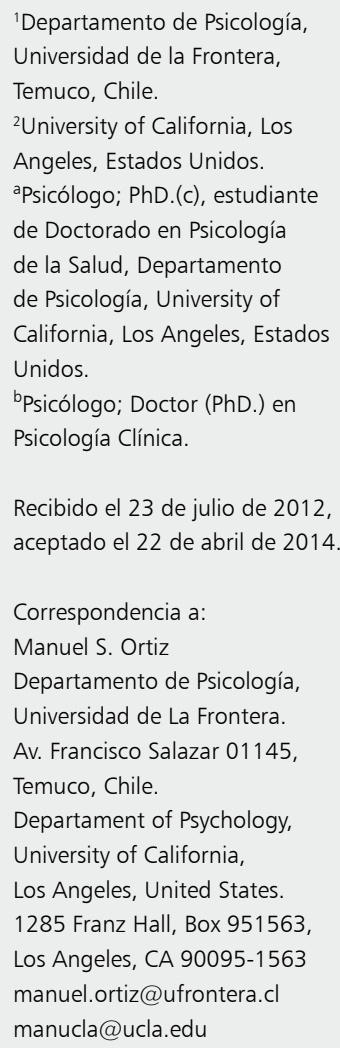

cuales pueden incrementar el riesgo de presentar síntomas depresivos ${ }^{6-10}$, baja adherencia al tratamiento ${ }^{11,12}$ y pobre control metabólico ${ }^{13}$.

El estrés psicológico puede ejercer sus efectos de modo directo o indirecto, ya sea interfiriendo la ejecución de las conductas pilares del tratamiento o bien por medio de la activación persistente del eje adrenérgico hipotalámico pituitario, generando una cascada de eventos fisiológicos que finalmente conllevan la liberación de glucocorticoides y por tanto, elevación de niveles de glicemia ${ }^{14,15}$. Diversos estudios han reportado la existencia de asociaciones entre estrés psicológico y control 
metabólico, sugiriendo que mayores niveles de estrés tenderían a asociarse con altos niveles de glicemia ${ }^{16-20}$. Sin embargo, estos estudios presentan ciertas limitaciones tales como haber considerado al estrés como un factor general desconociendo la multidimensionalidad del constructo, utilizar escalas de autorreporte que tienden a cuantificar la percepción de eventos estresantes o permiten al participante definir que es lo que él entiende como estresante, o bien establecer la relación entre estrés y control metabólico en un momento dado del tiempo, por medio de estudios transversales.

El presente estudio, utiliza un diseño longitudinal y una escala específica para medir estrés en pacientes diabéticos, desarrollada por Polonsky et $\mathrm{al}^{21}, \mathrm{y}$ que permite identificar un factor general y 4 subfactores: estrés afectivo/emocional, estrés con el médico, estrés relacionado con el régimen de tratamiento y estrés interpersonal.

De acuerdo a los postulados de Polonsky et $\mathrm{al}^{21}$, se podría esperar altos niveles de estrés emocional como consecuencia del diagnóstico y de vivir con diabetes. Asimismo, altos niveles de estrés con el médico y estrés con el régimen de tratamiento podrían asociarse con las demandas que impone el tratamiento. En la misma dirección, altos niveles de estrés interpersonal como consecuencia del diagnóstico y de vivir con diabetes DM1 podrían interferir con las relaciones sociales del adolescente.

El objetivo del presente estudio es determinar la asociación longitudinal entre cuatro dominios del estrés psicológico: estrés emocional, estrés relacionado con el régimen de tratamiento, estrés con el médico y estrés interpersonal con el control el control metabólico de adolescentes DM1.

\section{Material y Método}

\section{Participantes}

Una muestra no probabilística e intencionada fue seleccionada desde los registros del Hospital Hernán Henríquez de Temuco, Chile. Veinte adolescentes DM1, con más de un año de diagnóstico y cuya edad fluctuó entre los 12 y 18 años asintieron/ consintieron en participar en el estudio.

De los 20 participantes, $60 \%$ corresponde al género masculino. El promedio de edad fue $15,15(\mathrm{DE}=3,8)$ y el promedio de años viviendo con diabetes fue 5,07 ( $\mathrm{DE}=3,5)$. En promedio los adolescentes reportaron realizar 3,6 glicemias diarias $(\mathrm{DE}=1,09)$ e inyectarse insulina diariamente 3,89 veces $(\mathrm{DE}=1,15)$. El valor de hemoglobina glicosilada promedio en la primera medición (tiempo 0) fue 9,23 ( $\mathrm{DE}=2,1)$, en la segunda (tiempo 1$)$ fue $8,79(\mathrm{DE}=1,74)$ y en la medición final (tiempo 2) fue 8,12 $(\mathrm{DE}=1,82)$. El promedio de las tres medidas de hemoglobina fue $8,71(\mathrm{DE}=1,74)($ Tabla 1$)$.

\section{Instrumentos}

Estrés. Para evaluar el estrés, se utilizó la Escala de Estrés para Diabéticos de Polonski ${ }^{21}$, (2005) cuya versión original cuenta con 17 ítems, agrupados en 4 factores (subescalas): subescala de estrés emocional (EB), subescala de estrés con el médico $(\mathrm{PD})$, subescala de estrés con el régimen de tratamiento $(\mathrm{RD})$ y la subescala de estrés interpersonal (ID). El formato de respuesta original de la escala es de 6 puntos, donde cada participante debe responder cuan problemático es cada uno de los ítems presentados. Sin embargo, la escala aplicada a los participantes de este estudio fue adaptada a un formato de respuesta tipo Likert de 5 puntos donde mayores puntajes indican mayores niveles de estrés, $(1=$ muy en desacuerdo y $5=$ muy de acuerdo) donde mayores puntajes indican mayores niveles de estrés. Este formato de respuesta fue considerado más familiar para los participantes y posiblemente podría garantizar una mejor comprensión y calidad de respuesta. La consistencia interna de la escala total, estimada con el coeficiente alfa de Cronbach fue 0,879. La confiabilidad para las subescalas fue la siguiente: estrés emocional $=0,888$; estrés con el médico $=0,78$; estrés con el régimen de tratamiento $=$ 0,906; estrés interpersonal $=0,681$.

Para los fines de este artículo se reportan sólo los puntajes de las cuatro subescalas. En una versión previa a este manuscrito se informaron resultados con el puntaje total de la escala ${ }^{13}$.

Control metabólico: A cada paciente se le practicaron 3 exámenes de hemoglobina glicosilada (HbAlc). La HbA1c provee una medida confiable de glicemia crónica y correlaciona bien con los riesgos de complicaciones a largo plazo ${ }^{22}$.

Covariables: Diversas variables tales como sexo, edad, años con diabetes, práctica de actividad física (sí/no), fueron auto-reportadas por los participan- 
tes e ingresadas a los modelos estadísticos como covariables.

\section{Procedimiento}

Este estudio adhirió a los principios éticos que dicta la American Psychological Association ${ }^{23}$. Cada participante y su padre/tutor (en caso de ser menores de edad) firmaron una carta de asentimiento y consentimiento informado. Detalles del estudio fueron explicados en forma oral y escrita. El carácter voluntario, anónimo y confidencial del estudio fue explicitado. Cada adolescente fue recompensado económicamente con 4.000 pesos chilenos por su participación en cada examen, procedimiento considerado estándar para estimular participación y reducir mortalidad en la muestra.

Los tres exámenes de $\mathrm{HbAlc}$ fueron practicados con un intervalo de 3 meses aproximadamente. El resultado de cada examen fue informado inmediatamente. Cada medición fue realizada con el equipo DCA 2000+ del Laboratorio Bayer.

\section{Análisis estadístico.}

Los datos fueron analizados con el software estadístico STATA 12.0. Se realizó análisis longitudinal de curvas de crecimiento (longitudinal growth curve modeling $)^{24}$, el cual permite un manejo flexible del tiempo y permite la no independencia de las medidas repetidas en cada sujeto. Asimismo, sumado a los tradicionales parámetros obtenidos de un análisis de regresión (constante y pendiente de regresión) este análisis permite estimar una constante aleatoria que captura la variabilidad inicial entre los sujetos (en nuestros modelos las diferencias individuales de los participantes en la medida inicial de $\mathrm{HbAlc}$ ) y pendientes aleatorias que capturan la variabilidad de los participantes en el cambio a través del tiempo (en nuestros modelos las trayectorias de HbAlc de cada participante). Sumado a lo anterior esta técnica permite que la constante y las pendiente de regresión correlacionen, indicando el grado de asociación entre la medida inicial de un individuo y su cambio en el tiempo. Por ejemplo, una correlación alta y negativa entre constante y pendiente indicaría que para un participante que presente altos niveles de HbAlc en la medida inicial, mayor sería su disminución de HbAlc en el tiempo (medidas 1 y 2 en nuestros modelos), en comparación con un paciente que presente niveles de hemoglobina glicosilada en torno al promedio.
En todos los modelos se incluyó una constante aleatoria y una pendiente para el tiempo, permitiendo además que ambos correlacionaran. Cada examen de HbAlc fue codificado como tiempo 0,1 y 2 . Las medidas de estrés y los exámenes de $\mathrm{HbAlc}$ (tiempo) fueron ingresados en todos los modelos. Todas las covariables fueron ingresadas en nuestros modelos así como también los términos de interacción entre el tiempo y los 4 dominios de estrés (tiempo X EB, tiempo X PD, tiempo X $\mathrm{RD}$, tiempo X ID). Se utilizó como criterios de significación estadístico un valor de probabilidad igual o menor a $0,05(\mathrm{p} \leq 0,05)$.

\section{Resultados}

\section{Trayectorias del control metabólico}

En el primer análisis únicamente se incluyeron los tiempos $(0,1,2)$ y las covariables con la finalidad de caracterizar las trayectorias de HbAlc. La constante estimada para el tiempo 0 fue $b=9,3$ y su variabilidad $s_{\text {constante }}=1,76$, indicando que el promedio de HbAlc en el tiempo 0 fue 9,3 y la variabilidad individual en torno al promedio fue 1,76 . El efecto del tiempo fue negativo y estadísticamente significativo $(b=-0,55[95 \% \mathrm{CI}=-0,85$; $-0,25])$ sugiriendo una tendencia a la disminución en los valores de hemoglobina a lo largo del estudio (Figura 1). Ninguna de las covariables fue estadísticamente significativa y por tanto no fueron incluidas en subsecuentes modelos.

Posteriores análisis fueron realizados inclu-

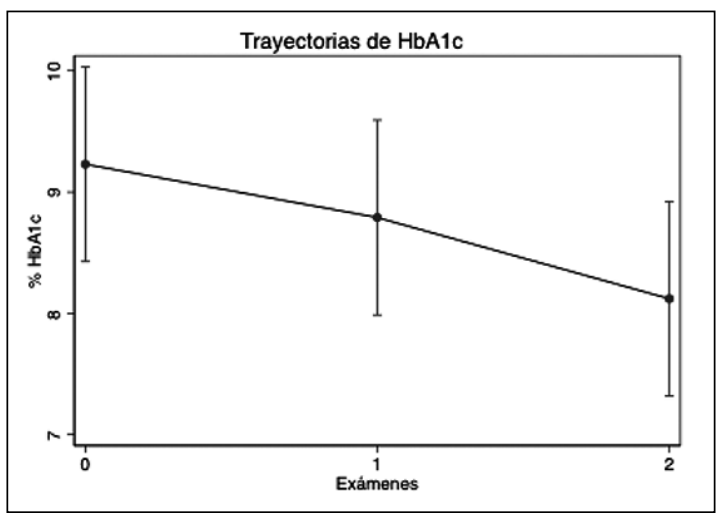

Figura 1. Trayectorias de HbA1c. En el gráfico se observa que independientemente de los niveles de estrés psicológico, el control metabólico tiende a la disminución a través del tiempo (asume una tendencia lineal negativa). 
Tabla 1. Características de los participantes

\begin{tabular}{|lc|}
\hline Variable & $\mathbf{n}[\%]$ o M [DE] \\
Edad (años) & $15,15[3,8]$ \\
Años viviendo con diabetes & $5,07[3,5]$ \\
Sexo (mujer/hombre) & $8 / 12[40 \% / 60 \%]$ \\
Actividad física (si/no) & $17 / 3[85 \% / 15 \%]$ \\
Cantidad glicemias diarias & $3,6[1,09]$ \\
Inyecciones diarias insulina & $3,89[1,15]$ \\
Estrés emocional & $12,45[4,97]$ \\
\hline Estrés con el médico & $8,6[4,05]$ \\
\hline Estrés con el régimen de tratamiento & $12,1[5,59]$ \\
Estrés interpersonal & 7 \\
\% HbA1c Tiempo 0 & $9,23[2,99]$ \\
\% HbA1c Tiempo 1 & $8,79[1,74]$ \\
\hline$\%$ HbA1c Tiempo 2 & $8,12[1,82]$ \\
\hline
\end{tabular}

yendo los dominios de estrés y sus respectivos términos de interacción (eg. Tiempo X EB), con la finalidad de estimar si cada uno de estos predijeron las trayectorias de HbAlc (Figura 2). El primer análisis incluyó el dominio de EB (Modelo 1 en Tabla 2). Tal como era esperable el EB predijo de manera estadísticamente significativa los niveles de HbAlc $(b=0,18$ [95\%CI = 0,02;0,33]), de modo tal que altos niveles de EB se asociaron con altos niveles de $\mathrm{HbAlc}$ en los tres exámenes realizados. El término de interacción tiempo X EB no fue estadísticamente significativo. En el modelo 2 el análisis fue efectuado con el dominio PD, sin embargo ni este ni su término de interacción con el tiempo predijeron trayectorias de HbAlc. En el tercer modelo se analizó el efecto del RD el cual se asoció positivamente con los niveles de $\mathrm{HbAlc}$ $(b=0,22[95 \% \mathrm{CI}=0,13 ; 0,35])$, sugiriendo que altos niveles de RB se asociaron con altos niveles
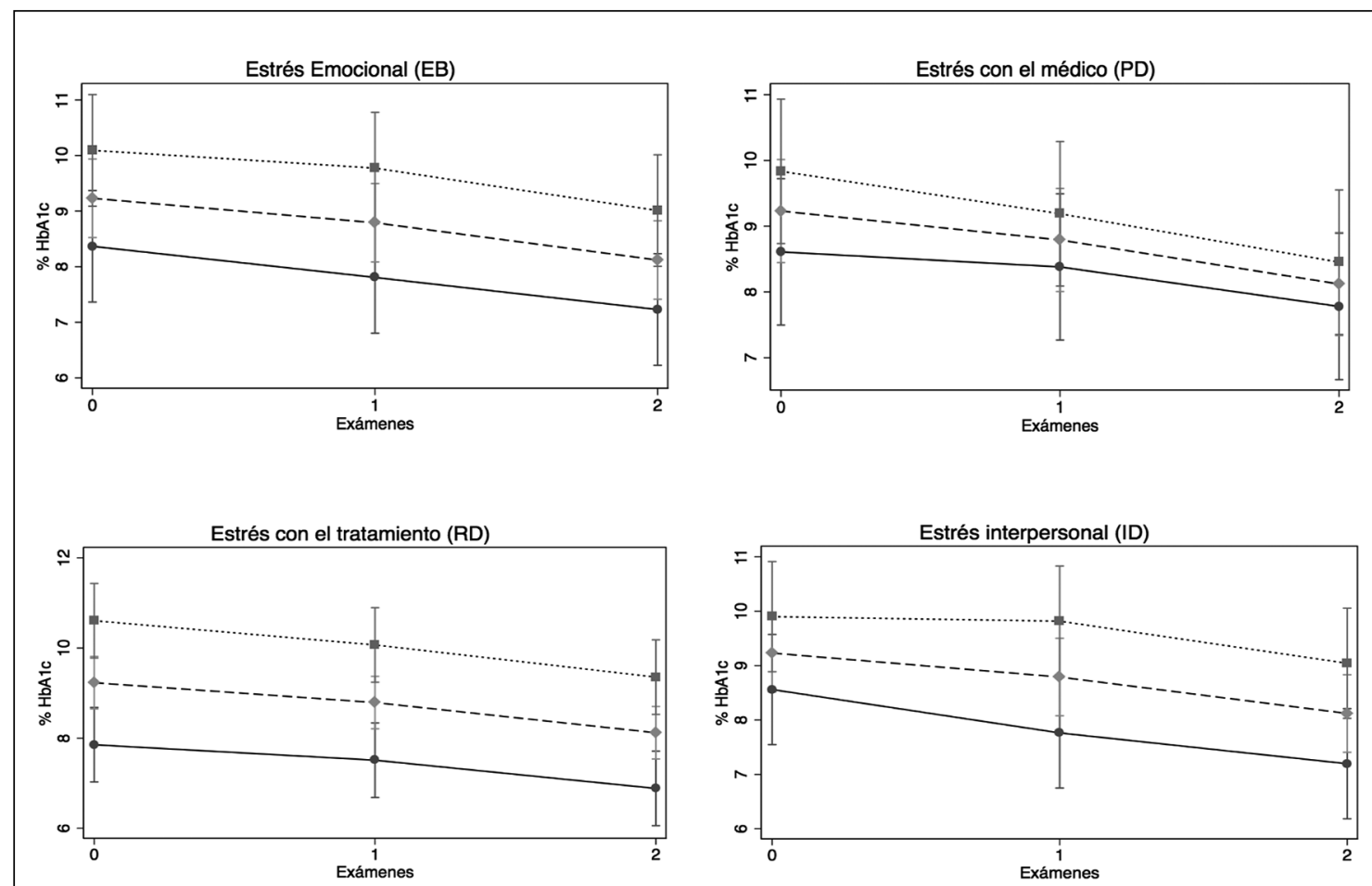

Figura 2. Dominios de estrés psicológico y trayectorias de HbA1c. Línea . . . . . . . = +1DE; Línea - - - - - - = Promedio; Línea $-1 \mathrm{DE}$. En todos los tiempos evaluados, participantes puntuando 1 desviación estándar (+1DE) por sobre el promedio en el dominio de estrés evaluado, presentan $\mathrm{HbA} 1 \mathrm{c}$ más elevadas que aquellos participantes puntuando 1 desviación estándar (-1DE) por bajo el promedio. 
Diabetes tipo 1: rol del estrés psicológico - M. S. Ortiz et al

Tabla 2. Asociación longitudinal entre los dominios de estrés y HbA1c

\begin{tabular}{|c|c|c|c|c|}
\hline & Modelo 1: EB & Modelo 2: PD & Modelo 3: RD & Modelo 4: ID \\
\hline Constante & $9,26^{* *}[8,52 ; 10,01]$ & $9,26^{* *}[8,46 ; 10,06]$ & $9,26^{* *}[8,67 ; 9,86]$ & $9,26^{* *}[8,49 ; 10,0]$ \\
\hline Tiempo & $-0,58 \quad[-1,39 ; \quad 0,21]$ & $-0,25 \quad[-0,94 ; 0,43]$ & {$[-1,11 ; 0,30]$} & $-0,85^{*} \quad[-1,60 ;-0,1]$ \\
\hline Estrés & $0,18^{*} \quad[0,02 ; 0,33]$ & $0,14 \quad[-0,05 ; 0,34]$ & $0,22^{* *}[0,13 ; 0,35]$ & $0,24^{\dagger} \quad[-0,01 ; 0,51]$ \\
\hline Tiempo X estrés & $0,00 \quad[-0,05 ; 0,06]$ & $-0,03 \quad[-0,10 ; \quad 0,03]$ & {$[-0,06 ; 0,04]$} & {$[-0,05 ; 0,14]$} \\
\hline$\sigma$ Constante & {$[1,03 ; 2,25]$} & {$[1,14 ; 2,42]$} & {$[0,71 ; 1,80]$} & {$[1,09 ; 2,35]$} \\
\hline$\sigma$ Tiempo & {$[0,09 ; 1,42]$} & {$[0,06 ; 1,59]$} & {$[0,08 ; 1,45]$} & {$[0,07 ; 1,55]$} \\
\hline$\sigma$ Tiempo $X$ estrés & $-0,55 \quad[-0,91 ; 0,31]$ & $-0,38 \quad[-0,87 ; \quad 0,48]$ & {$[-0,90 ; 0,38]$} & {$[-0,09 ; 0,49]$} \\
\hline$\sigma$ Residuo & {$[0,59 ; 1,11]$} & {$[0,59 ; 1,11]$} & {$[0,59 ; 1,11]$} & {$[0,59 ; 1,11]$} \\
\hline
\end{tabular}

Modelo 1 incluye como predictor el estrés emocional (EB). Modelo 2 incluye como predictor el estrés con el médico. Modelo 3 incluye como predictor al estrés con el régimen de tratamiento. Modelo 4 incluye como predictor al estrés interpersonal. ${ }^{* *} p \leq 0,01 ;{ }^{*} p \leq 0,05 ;{ }^{\dagger} p \leq 0,10$.

de HbA1c en los tres exámenes realizados. La interacción tiempo X RD no fue estadísticamente significativa. Finalmente, el análisis en el cuarto modelo fue ejecutado con el dominio de ID y su respectivo término de interacción, obteniéndose un efecto marginalmente significativo $(\mathrm{p}=0,06)$ para el ID. Adicionalmente fue encontrado un efecto significativo para el tiempo $(b=-0,85$ $[95 \% \mathrm{CI}=-1,60 ;-0,1])$, sugiriendo que aquellos participantes que tienen altos niveles de estrés interpersonal tienden a disminuir sus niveles de HbA1c a través del tiempo. La interacción tiempo $X$ ID no fue estadísticamente significativa.

\section{Discusión}

El presente estudio tuvo por finalidad determinar la asociación longitudinal entre cuatro dominios del estrés psicológico con el control metabólico de pacientes adolescentes DM1.

De los cuatro dominios de estrés estudiados, se ha encontrado evidencia que tanto el EB y el $\mathrm{RD}$ se asociaron positivamente con la $\mathrm{HbAlc}$ en todos los tiempos estudiados, es decir aquellos participantes que presentaron valores elevados de estrés emocional y de estrés con el régimen de tratamiento tendieron a presentar concentraciones altas de HbA1c. Similar resultado se encontró con el ID, aunque esta asociación fue marginalmente significativa. Independientemente de los niveles de estrés, la HbAlc asumió una tendencia lineal negativa y estadísticamente significativa, lo cual implica que hubo un descenso global de los valores de HbAlc durante los tres tiempos estudiados.

Los resultados obtenidos están en línea con estudios previos que sugieren que el estrés psicológico se asocia con pobres resultados del control metabólico en adolescentes DM1 ${ }^{1,25}$, sin embargo, el resultado es interesante pues permite diferenciar dominios específicos del estrés psicológico que se asocian al control metabólico y descartar otros. Es así como no es sorprende haber encontrado que en todos los tiempos medidos, aquellos pacientes que presentaron mayor EB y mayor RD tendieron a tener HbAlc más elevadas. En efecto, el diagnóstico de la DM1 implica que el adolescente ejerza grandes modificaciones en sus hábitos, ajustes en la dieta y asuma nuevos patrones conductuales. Es posible que todos estos cambios, sumados a la etapa evolutiva generen altos niveles de estrés. Asimismo, adherir al nuevo plan alimenticio, contar carbohidratos y ajustar dosis de insulina ha sido identificado como un factor que impone grandes desafíos a pacientes diabéticos y en especial a adolescentes, quienes se caracterizan por ser impulsivos y tener altas probabilidades de involucrarse en eventos sociales con sus grupos de pares, en los cuales el foco de la actividad es la alimentación ${ }^{26}$. Aún más y pese a que en este estudio hubo una asociación marginalmente significativa entre el ID y HbAlc, este resultado es teóricamente esperable pues las demandas que impone la DM1 permean todas las actividades del adolescente incluyendo 
sus interacciones sociales, pudiendo generar elevados niveles de estrés interpersonal.

La no asociación del PD y el control metabólico es un resultado no esperado, pues en muchas ocasiones el médico puede ser visto como un agente que ejerce control externo sobre el adolescente, limitando la independencia del mismo y generando estrés psicológico. En este sentido parece muy importante para futuros estudios indagar en las percepciones que los adolescentes tienen sobre el equipo médico y analizar la relación médico-paciente, aspecto que resulta crítico en la construcción de una alianza terapéutica que beneficie al paciente. $\mathrm{Al}$ respecto, Kyngas, Hentinen y Barlow ${ }^{27}$ reportaron que adolescentes DM1 con buena adherencia al tratamiento tienden a reportar las acciones del médico como no autoritarias (considerando la opinión del paciente) y no rutinarias (la opinión del paciente es considerada en las recomendaciones que el médico provee y éstas son más bien flexibles).

Un resultado interesante en este estudio guarda relación con que el control metabólico entre el tiempo 0,1 y 2 , independientemente de los niveles de estrés psicológico, asumió una tendencia lineal negativa. Posibles explicaciones para esta tendencia negativa, podrían ser el efecto del feedback inmediato. El efecto del feedback inmediato ha sido reportado como una importante herramienta para mejorar el control metabólico de pacientes diabéticos ${ }^{28}$. Cagliero ${ }^{29}$ han reportado que el este tipo de feedback podría tener efectos beneficiosos para el posterior control metabólico a los 6 meses y 12 meses de seguimiento.

La presencia de efectos principales para el EB, RD y ID (este último marginalmente significativo), resaltan el rol que el estrés psicológico tiene sobre la HbAlc. El estrés psicológico puede tener efectos directos o indirectos sobre el control metabólico, ya sea alterando la adherencia a las conductas pilares del tratamiento de la DM1, o bien ejerciendo su efecto por medio de la activación crónica del eje adrenérgico hipotalámico pituitario ${ }^{14,15}$. Lamentablemente, una limitación de este estudio es que no permite identificar cual de estos mecanismos es el que da cuenta del efecto del estrés, y será interesante en futuros estudios incluir medidas de cortisol que indiquen activación crónica del eje adrenérgico hipotalámico pituitario o bien medidas de autorreporte de comportamiento adherente.
Otra limitación de estudio fue su tamaño muestral $(n=20)$, lo cual invita a ser cautelosos en la interpretación de estos resultados y se sugiere replicar este estudio con una muestra de mayor tamaño y de mayor representatividad nacional (inclusión de pacientes diabéticos tipo 2 y pacientes de otras regiones, etc.). Pese a lo anterior, se debe destacar que la técnica de análisis empleada en este estudio (growth curve modeling) es una técnica adecuada para el análisis de datos longitudinales y que además de las ventajas ya comentadas, garantiza mayor potencia estadística ${ }^{24}$.

Finalmente, la identificación de diferentes dimensiones del estrés psicológico asociadas con el control metabólico, representa una ventaja por sobre estudios que han identificado un factor general de estrés, pues permite identificar dominios críticos que podrían ser objeto de intervenciones específicas.

\section{Referencias}

1. Helgeson V, Escobar O, Siminerio L, Becker D. Relation of Stressful Life Events to Metabolic Control Among Adolescents With Diabetes: 5-Year Longitudinal Study. Health Psychol 2010; 29: 153-9.

2. Holmbeck G, Friedman D, Abad M, Jandasek B. Development and psychopathology in adolescence. En Wolfe D, Mash E. Editores, Behavioral and emotional disorders in adolescents. New York: The Guilford Press; 2006. p. 21-55.

3. Byrne D, Davenport SC, Mazanov J. Profiles of adolescent stress: The development of the Adolescent Stress Questionnaire (ASQ). J Adolesc 2007; 30: 393-416.

4. Byrne DG, Mazanov, J. Adolescent stress and future smoking behavior: A prospective investigation. J Psychosom Res 2003; 54: 313-21.

5. Ge X, Lorenz F, Conger R, Elder G, Simons R. Trajectory of stressful life events and depressive symptoms during adolescence. Dev Psychol 1994; 30: 467-83.

6. Whittemore R, Kanner S, Singleton S, Hamrin V, Chiu J, Grey M. Correlates of depressive symptoms in adolescents with type 1 diabetes. Pediatr Diabetes 2002; 3: 135-43.

7. Mcgrady E, Hood K. Depressive symptoms in adolescents with type 1 diabetes: Associations with longitudinal outcomes. Diabetes Res Clin Pract 2010; 88: 35-7.

8. Grey M, Whittemore R, Tamborlane W. Depression in type 1 diabetes in children: natural history and correlates. J Psychosom Res 2002; 53: 907-11.

9. Hood K, Huestis S, Maher A, Butler D, Volkening L, 
Laffel L. Depressive symptoms in children and adolescents with type 1 diabetes. Diabetes Care 2006; 29: 1389-11389.

10. Mcgrady M, Laffel L, Drotar D, Repaske D, Hood K. Depressive symptoms and glycemic control in adolescents with type 1 diabetes: mediational role of blood glucose monitoring. Diabetes Care 2009; 32: 804-6.

11. Patton S. Adherence to Diet in Youth with Type 1 Diabetes. J Am Diet Assoc 2011; 111: 550-5.

12. Ortiz M, Ortiz E. Adherencia al tratamiento en adolescentes diabeticos tipo 1 chilenos: Una aproximacion psicologica. Rev Med Chile 2005; 133: 307-13.

13. Ortiz M. Estabilidad de la adherencia al tratamiento en una muestra de adolescentes diabeticos tipo 1. Ter Psicol 2008; 26: 71-80.

14. Miller G, Chen E, Zhou E. If it goes up, must it come down? Chronic stress and the hypothalamic-pituitaryadrenocortical axis in humans. Psychol Bull 2007; 133: 25-45.

15. Sapolsky R, Romero M, Munck A. How Do Glucocorticoids Influence Stress Responses? Integrating Permissive, Suppressive, Stimulatory, and Preparative Actions. Endocr Rev 2000; 21: 55-89.

16. Guthrie D, Bartsocas C, Jarros-Chabot P, Konstantinova M. Psychosocial issues for children and adolescents with diabetes: Overview and recommendations. Diabetes Spectr 2003; 16: 7-12.

17. Adriaanse M, Pouwer F, Dekker J, Nijpels G, Stehouwer $\mathrm{C}$, Heine R, et al. Diabetes-related symptom distress in association with glucose metabolism and comorbidity: the Hoorn Study. Diabetes Care 2008; 31: 2268-70.

18. Fisher L, Mullan J, Skaff M, Glasgow R, Arean P, Hessler D. Predicting diabetes distress in patients with Type 2 diabetes: a longitudinal study. Diabet Med 2009; 26: 622-7.
19. Fisher L, Skaff M, Mullan J, Arean P, Glasgow R, Masharani U. A longitudinal study of affective and anxiety disorders, depressive affect and diabetes distress in adults with Type 2 diabetes. Diabet Med 2008; 25: 1096-101.

20. Ortiz M, Ortiz E, Gómez D, Gatica A. Factores Psicosociales Asociados a la Adherencia al Tratamiento de la Diabetes Mellitus Tipo 2. Ter Psicol 2011; 29: 5-11.

21. Polonsky W, Fisher L, Earles J, Dudl R. Assesing psychosocial distress in diabetes: Development of the diabetes distress scale. Diabetes Care 2005; 28: 626-31.

22. International Expert Committee. International Expert Committee report on the role of the A1C assay in the diagnosis of diabetes. Diabetes Care 2009; 32: 1327-34.

23. American Psychological Association. Ethical principies of psychologists and code of conduct. Am Psychol 1992: 47: 1597-611.

24. Singer JD, Willett JB. Applied Longitudinal Data Analysis: Modeling Change and Event Occurrence. New York, Oxford Univ. Press, 2003.

25. Lloyd C, Smith J, Weinger K. Stress and diabetes: A review of the links. Diabetes Spectr 2005; 18: 121-7.

26. Borus J, Laffel L. Adherence challenges in the management of type 1 diabetes in adolescents: prevention and intervention. Curr Opin Pediatr 2010; 22: 405-11.

27. Kyngäs $H$, Hentinen M, Barlow J. Adolescents' perceptions of physicians, nurses, parents and friends: help or hindrance in compliance with diabetes self-care. J Adv Nurs 1998; 27: 760-9.

28. Delamater A. Clinical use of hemoglobin Alc to improve diabetes management. Clin Diabetes 2006; 24: 6-8.

29. Cagliero E, Levina E, Nathan D. Immediate feedback of HbA1c levels improves glycemic control in type 1 and insulin-treated type 2 diabetic patients. Diabetes Care 1999; 22: 1785-9. 